\title{
The Norwegian research programme on advanced robotic systems
}

\begin{abstract}
OLAV EGELAND $\dagger$
Keywords: Robotics, ROV, telerobotics, sensory robot control, subsea robotics, robotics overview.

The Norwegian research programme on advanced robot systems has bèen focused on sensory control of robots for industrial applications and telerobotics for underwater operations. This paper gives an overview of experimental work and ongoing research. An exciting area in sensory control is visual servoing where camera images at video rate are used to grasp moving objects. Also compliant motion in partially unknown environments is a research topic. New robot control systems have been developed to apply sensory control to robotic manipulators at an acceptable sampling rate. In telerobotics the main work has been on the combination of remote control and local sensory loops in the manipulator. Also in this case visual servoing and force control are important. The generation and updating of a world model used in a graphic display of the worksite using sensory information has been tested in combination with large delay times in the communication channel. The use of visual and acoustic data for the control of remotely operated vehicles and autonomous underwater vehicles is studied for use in robotic systems. Light-weight robot manipulators with redundant degrees of freedom and high performance joints are being designed for mobile robot applications.
\end{abstract}

\section{Introduction}

The first Norwegian industrial robot was made by Trallfa in 1966 and was commercially available in 1969 . This was a spray-painting robot which eliminated the need for workers in the health-damaging spray-painting process. Since then several Norwegian companies have been working with inventive designs for industrial manipulators.

There is much economic activity on the Norwegian Continental Shelf, dominated by oil exploration and production. The new oil-fields are at water depths of $350 \mathrm{~m}$ and more, and it is necessary to use telerobotics for maintenance tasks in the hostile deepwater environment.

A large part of Norway is mountainous and tunnels are important in road construction. This created the need for computer controlled rock-drilling machines.

The good tradition in industrial robotics, and the needs of the oil industry make it interesting for Norway to develop advanced robotic systems. Also Norway's interest in protecting workers from health hazards gives a motivation for developing robotic systems that can dispense with human work in hostile environment. This has lead to research projects sponsored by the Royal Norwegian Council for Industrial and Scientific Research (NTNF) where industrial companies have cooperated with the Norwegian Institute of Technology and Norwegian research institutions.

This paper gives an overview of Norwegian research activity on advanced robotics.

Received 10th March 1991.

$\dagger$ Division of Engineering Cybernetics, The Norwegian Institute of Technology, N-7034 Trondheim, Norway.

This paper was presented at the '91 International Symposium on Advanced Robot Technology ('91 ISART), 5-7 March 1991, Tokyo, Japan. Reprinted with permission from the Japan Industrial Robot Association. 


\section{Industrial robotics}

Industrial robotics has reached a certain maturity, but there are interesting tasks that cannot be executed with present day technology, especially due to the lack of sensors and dexterity. In the Center for Robotic Research at the Norwegian Institute of Technology sensory control and the use of redundant degrees of freedom have been investigated for use in next generation industrial robots.

Industrial robot controllers are designed to track a predefined reference trajectory, and the interface to external signals for feedback from sensors in the end-effector is usually too slow to facilitate high performance sensory control. As a consequence of this, relatively few research laboratories have adequate experimental equipment for experiments in sensory control of industrial robots.

At the Norwegian Institute of Technology sensory control systems have been developed with an interface at the Cartesian position level with a sampling frequency of $100-500 \mathrm{~Hz}$. This has been done for the ABB Trallfa TR400 and Multicraft $560 \mathrm{in}$ cooperation with the companies producing the robots. The control systems are VMEbased, and Motorola microprocessors are used for control at the Cartesian level. These control systems give a very good experimental environment.

\section{Telerobotics}

Telemanipulation has been extensively used to perform work in hostile environments. Developments in recent years have been to include more intelligence into the control system to get shared control, or telerobotics which is a combination of robotics and teleoperation. Useful techniques in telerobotics are:

Sensor-based telerobotics which is similar to sensor-based robot control. With this technique the end effector motion is divided into sensor-controlled and teleoperated directions. This is useful for inspection tasks where position deviation sensor can be used to achieve a constant distance and orientation with respect to the surface to be inspected, while the operator specifies the motion tangentially to the surface. This technique has been tested out in preliminary experiments.

Preprogrammed subtasks can be used to perform standard operations like pegin-hole insertion. In this mode the operator gives a gross positioning of the end effector, while the subtask is executed automatically using sensors.

A problem in sub-sea telerobotics is the time delay in the communication channel between operator and manipulator. This is especially difficult when force-reflection is used which in many casese causes instability. New solutions to this problem based on passivity theory (Anderson and Spong 1989) have been studied at the Norwegian Institute of Technology (Hovland 1990).

\section{Sensory control systems}

\subsection{Visual servoing}

The use of camera measurements in the feedback loop has been investigated for robot control (Egeland et al. 1990, Skofteland and Hirzinger 1991). 50 or $60 \mathrm{~Hz}$ sampling of a scene can be achieved with standard video cameras, and if the robot vision algorithm is fast enough the measurement can be used for tracking moving objects. It is clear that even with the powerful processors that are now availble the robot vision must be fairly simple to run at $50 \mathrm{~Hz}$. However, in many robot applications the 
object to be tracked has a known geometry, and this reduces the computational requirements dramatically (Dickmanns and Gräfe 1988).

Successful experiments on visual servoing of an industrial robot at $50 \mathrm{~Hz}$ have been done, and experiments with stereo vision will be done in the near future.

\section{Force control}

Force control of manipulators has been extensively studied in the research literature (see for example, Whitney 1987), but high quality force control systems have not to our knowledge become commercially available for industrial manipulators. This is to a large extent due to the lack of fast interfaces on the Cartesian level. The limited interest in industry with regards to force control of manipulators is also due to disappointing results in early experiments with inadequate interfaces.

Much attention has been given to the grinding and deburring in Norwegian research and development in robotic force control. A commercial grinding system available for the Norwegian Multicraft 560 robot was developed by the research foundation SI in Oslo in cooperation with the Multicraft company. The system adjusts the tangential velocity of the grinding tool as a function of the contact force which gives a substantial improvement in grinding operations.

Other systems have been developed and experimentally tested at the Norwegian Institute of Technology. Grinding under force control has been tested out with good results, and contour following has been used for programming of grinding and deburring tasks (Thomessen and Lien 1990).

\section{Singularity handling}

The inverse kinematic transformation for end-effector coordinates to joint coordinates has singularities where the manipulator Jacobian loses rank, and the usual resolved rate solution becomes undefined. This is experienced in the form of very high joint velocities and large control deviations close to the singular configurations. In conventional industrial robotics using preprogrammed trajectories this is not a serious problem as it is possible to use joint space interpolation in the neighbourhood of singularities or to plan trajectories that do not go through singularities. However, in sensory control or in joy-stick programming the manipulator may enter singular configurations under Cartesian control, and it is important to have some kind of singularity handling.

Our work in this field has to some extent been based on the damped least-squares solution as proposed in Nakamura and Hanafusa (1986). The main contribution of our work has been on the selection of a damping factor which gives acceptable joint velocities and at the same time a good accuracy close to singularities. Techniques for specifying if the control deviation due to a singularity should appear in translation or in rotation have also been studied in experiments, and the results are reported in Egeland et al. $(1990,1991)$.

\section{Manipulator design}

\subsection{Vehicle/manipulator systems - Redundant degrees of freedom}

Vehicle/manipulator systems are interesting in many applications like underwater manipulation systems. To achieve efficient operation for this type of system it is useful to make an integration design of the vehicle and manipulator control system. Then it is possible to use the vehicle for gross positioning, while the manipulator gives fast and accurate motion of the end effector (Sagli and Egeland 1991). 
To increase the manipulator workspace in the presence of obstacles and with wrist singularities, the inclusion of a seventh joint in the shoulder of a standard PUMA type of geometry has been studied, and methods for redundancy resolutions have been proposed (Egeland et al. 1989, 1991). The redundancy resolution has been tested in experiments with an eight-joint spray-painting robot from ABB Trallfa and a simple four joint planar mechanism.

\subsection{Manipulators with link and joint flexibility}

The finite element programme system FEDEM (Finite Element Dynamics in Elastic Mechanisms) is a general simulation system for flexible mechanisms with an option for including control modules. FEDEM was developed at the Mechanical department at the Norwegian Institute of Technology in cooperation with the associated research foundation SINTEF (Sivertsen et al. 1990). The program system is based on a nonlinear finite element formulation utilizing component mode synthesis (CMS) for model reduction prior to the assembling of the mechanisms model. A static equilibrium solution may be computed before the dynamic integration of the mechanism motion. An eigenvalue analysis option is available for specified positions during integration. Stress results for the mechanism links can be calculated for specified time steps and presented graphically. A separate module is used for animation of mechanism motion or for the generation of video animation. The animation can also show the deflection of the parts during mechanism motion and even the stress distribution as colour fringes. For pre- and post-processing of element data, FEDEM has an interface to the commercial programmes PATRAN, FEMGEN/FEMVIEW and SUPERTAB. The simulation is very efficient on the one hand due to the algorithms for matrix operations and solution of equations, and on the other hand due to the techniques for CMS model reduction and data handling. The modeling tools consist of an extensive FEM library of elements, joints, linear and nonlinear springs and dampers, gravity forces, external loads and a number of other modeling options based on the finite element techniques. Control elements can be selected from a library that can easily be extended. A number of industrial simulation applications have been carried out within the field of robotics, offshore mechanisms, automobiles and mechanisms for space deployment.

From the early seventies general computer programmes for simulation of rigid body mechanism kinematics and dynamics have been developed, and several systems (such as ADAMS, IMP and later DADS) became commercially available. Extending the simulation techniques to include flexibility of the links and the influence of the automatic control system is an increasing demand from industry, especially aerospace companies, but also companies in robotics, offshore and car manufacturing. The systems mentioned so far are based on traditional rigid body theory, and attempts so far to include link flexibility into such systems have not proved efficient and practical and have limited applicability. For instance, flexible telescopic joints cannot be simulated by any of these systems.

Some of the systems mentioned above have options to include control elements, but positions, velocities, accelerations and forces are only given in joints, springs, dampers and points as results, and the dimensioning which depends on deformation and stress distribution in the links must be found in a separate operation.

These problems are solved in the FEDEM system which takes the flexibility of the individual parts of the mechanism into account, and the resulting simulation is of very good accuracy even for complex two and three dimensional mechanisms. 


\section{Subsea operations}

Maintenance and inspection of oil rigs have to a large extent been done by divers. This is relatively expensive because of the need for support vessels and the safety requirements. There have been numerous accidents because of the harsh working conditions for the divers. The most recently developed oil fields have water depth of $350 \mathrm{~m}$ and more, and this makes it expensive and difficult to use divers.

Remotely operated vehicles (ROVs) with telemanipulators are commercially available where the operator controls the manipulator motion in teleoperation mode. This task can be very demanding for the operator who must control all degrees of freedom of the manipulator, and in some cases also the vehicle degrees of freedom.

\subsection{Nondestructive testing of oil rigs}

The subsea structure of an oil rig must be inspected for cracks which may appear on modal weld-seams. Prior to inspection the sea growth must be removed from the weldseams. The inspection can be done for steel structures by scanning an eddy-current probe over the weld-seams of interest. This can be done by divers at moderate depths, and teleoperation is also used.

An interesting approach is to use telerobotics with additional sensors and a CAD model of the weld-seam to achieve removal of growth and a subsequent scanning of the seam, while the operator supervises the process. Preliminary experiments have been performed to test the use of position deviation sensor to scan a weld-seam under water. Underwater vehicles and manipulators are available for this task, and the integration of sensor and controllers with existing hardware will hopefully be done in the near future.

\subsection{Remotely operated subsea machine tool system}

The research foundation SINTEF at the Norwegian Institute of Technology has together with the Norwegian State Oil Company Statoil developed a concept for a remotely operated subsea machining tool system for deep-water precision machining and inspection (Husebye et al. 1990). The system is designed for water depths down to $400 \mathrm{~m}$. The machine tool system is operated through a dedicated Computer Numerical Control System (CNC) from a monohull vessel or rig.

The machine tool system is lowered on guide wires and is designed for remachining and documentation of various damages on seal surfaces on wellheads, permanently installed flanges and multibore connector hubs at the sea floor. In this way production delays and losses can be avoided, and the risk of abandonment of wells is reduced.

Parts of the remotely operated subsea machine tool system have been built, and the rest is developed to a stage so that a complete prototype system can be built and demonstrated.

The background for the project is that Statoil is the major operator of oil and gas fields on the Norwegian Continental Shelf, and is operating the Statfjord Gullfaks and Tommeliten fields. Company operations currently include operatorship of 12 subsea oil and gas wells, which is expected to increase to 70 by 1998 . Partner operations add the total number of subsea wells with significant Statoil ownership to approximately 150 by year 2000 .

Damages to wellhead sealing surfaces and flanges have occurred, and this has clearly shown the need for a diverless subsea machining system. As new fields are developed on water depths down to $350-400 \mathrm{~m}$ as opposed to today's fields at $100-150 \mathrm{~m}$ depths the availability of such a system becomes increasingly important. 


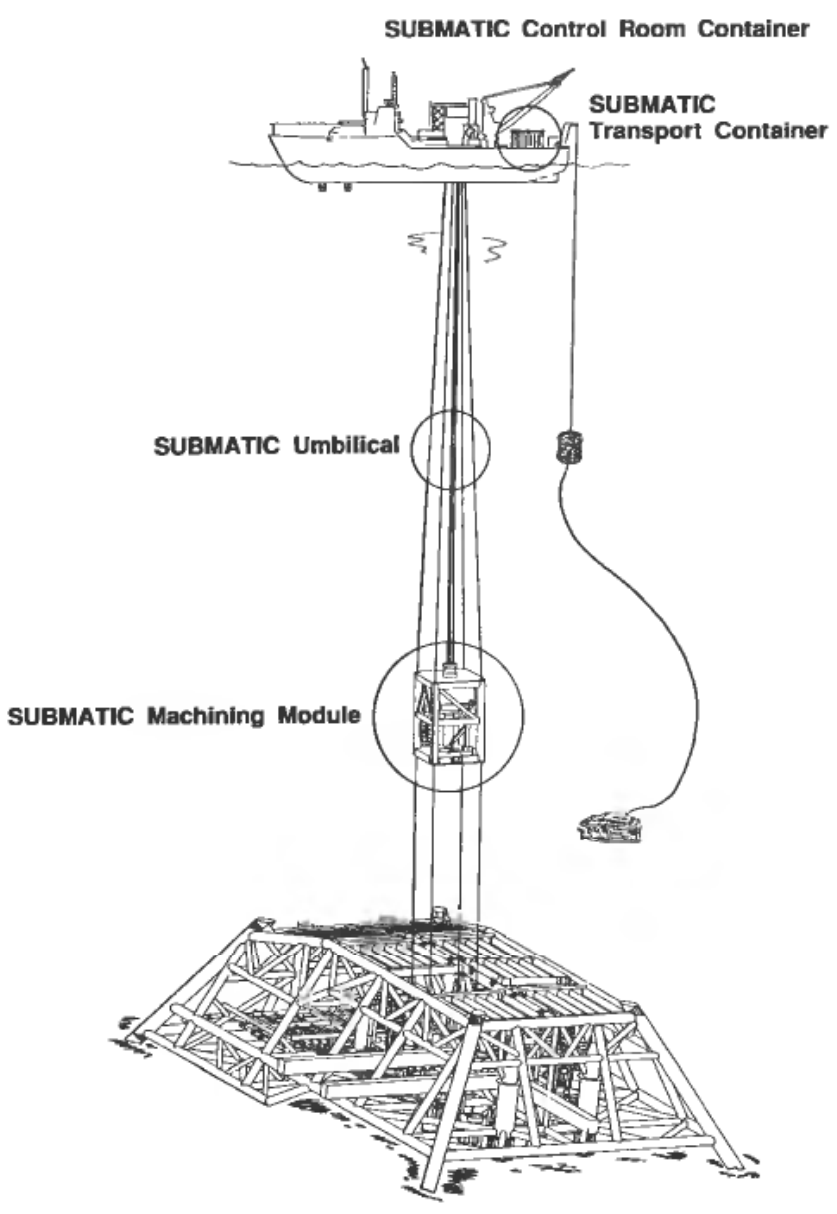

Figure 1. Remotely operated machining system.

Costs of developing a typical Norwegian producing well is in the range of 40-50 million US dollars. The incentive to avoid abandonment of these high-cost, highproducing wells is of course very high.

The system which is called SUBMATIC is shown in Fig. 1.

The machining module consists of the following parts:

Machine tool unit (Four axis CNC machining centre).

Main system structure with clamping unit.

Manipulator with control system, measurement unit and auxiliary tools and components.

The height of the SUBMATIC machining module is $4.5 \mathrm{~m}$, and the weight is 20 tons.

\subsection{Autonomous underwater vehicles}

An autonomous underwater vehicle (AUV) is an unmanned underwater vehicle which is capable of independent operation for extended periods of time. The vehicle has no connection to operators or support systems on the surface. This means that the vehicle must be autonomous with respect to power and control. In particular it means 
that AUVs must be able to collect data on the environment and interpret these data to create an internal understanding of the environment and the vehicle state in relation to the environment.

The level of autonomy may differ depending on the complexity of the environment, the vehicle and the mission. Higher degree of complexity requires higher degree of autonomy.

Remotely operated vehicles (ROVs) are completely controlled by an operator on the surface. The main difference between the AUV and the ROV is the ability of the AUV to navigate and control itself and its independence of a power system on the surface. The conversion of an ROV to an AUV requires the development of the following components:

Power System. The AUV needs energy for propulsion and electronics. The energy can be produced on board or stored in batteries.

Sensor System. The AUV must collect data concerning the environment and use the data to modify or replan its mission. The most useful underwater sensors are sonar and cameras.

Perception System. The sensor data must be converted to a format suitable for reasoning.

Decision System. The output from the perception system must be compared to the mission plan and other types of stored knowledge (reasoning). The result of the comparison is used for modification or replanning the mission (decision). Old measurements and decisions can be stored for later reference (learning).

System Structure. All the above components must be integrated into one system.

The following research problems in AUV technology are investigated:

Sensor-based world mapping is done using sparse and dense sonar data. The world mapping is used to determine the vehicle position using an a priori map. The sensor data is also used to update the map.

Object classification is used for automatic recognition of different types of objects on the ocean floor. This has been used for the tracking of pipelines (Hallset 1991) which is currently done with video cameras, but the use of laser scanners is also investigated.

System structures for AUV have been developed (Rødseth 1990). The computer system integrates many functional modules with very different requirements with respect to real time performance and data structures. The proposed solution includes guidelines for organization of modules, communication between modules and methods for error detection and correction.

Adaptive and robust controllers are needed to adjust for changing hydrodynamic parameters (Fossen and Sagatun 1991) and in addition reconfigure the controller structure in the event of a thruster failure.

Image compression and transmission is studied to facilitate the transfer of video and sonar data over a narrow-band communication link like a hydro-acoustic communication system.

\subsection{The Simrad vehicle}

The first autonomous vehicle built in Norway was the Simrad vehicle made in cooperation between the Norwegian Institute of Technology, SINTEF and Simrad Subsea a.s. (Kleppaker et al. 1986). The vehicle was battery powered and controlled through a hydro-acoustic link. The vehicle had enough autonomy to perform simple waypoint planning. It used a transponder-based navigation system. 


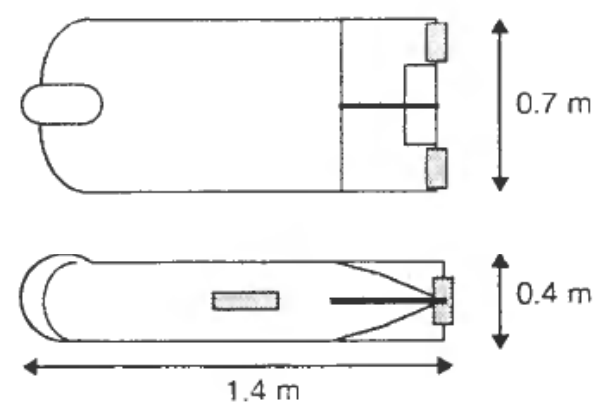

Figure 2. The Simrad vehicle.
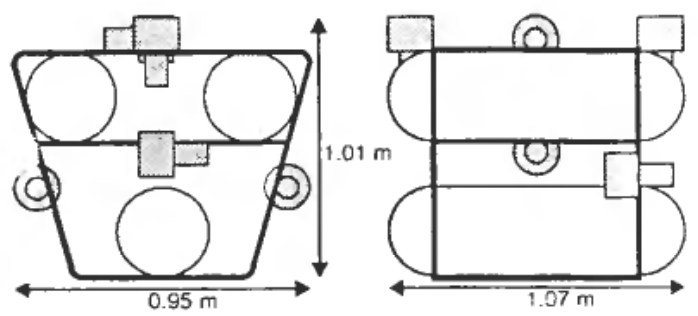

Figure 3. The NEROV vehicle.

The main data of the system was

$\begin{array}{ll}\text { Max depth } & 500 \mathrm{~m} \\ \text { Max speed } & 4-5 \mathrm{knots} \\ \text { Endurance } & 5 \text { hours at } 1.5 \text { knots } \\ \text { Weight } & 250 \mathrm{~kg} \\ \text { Range } & 700 \mathrm{~m} \text { (acoustic link) }\end{array}$

The vehicle is shown in Fig. 2. It had two thrusters astern and one side thruster. Motion in the vertical direction was controlled with control surfaces and a buoyancy compensation system. Successful sea test were done in 1987.

\subsection{NEROV-The Norwegian Expermerimental ROV}

NEROV is an experimental underwater vehicle built at the Norwegian Institute of Technology (Sagatun and Fossen 1991). The vehicle which is shown in Fig. 3 has six thrusters which can accelerate the vehicle in six degrees of freedom, and is equipped with position, orientation and angular velocity sensors for position and velocity control. The system has a powerful on-board computer system based on Motorola $68020 / 68881$ processors which makes it possible to run advanced control algorithms. The vehicle is powered by batteries which means that it is energy-autonomous, but it will have a coaxial communication cable for remote control. At a later stage it is planned to run experiments with fully autonomous operation.

In the first phase of this project the use of model-based control and adaptation will be tested out for position and velocity control of the vehicle with special emphasis on compensation for current and wave-induced disturbances. 
The vehicle is built of standard off-the-shelf components, and has a highly modular design. It is planned to use a modified version of the vehicle as a platform for an advanced underwater manipulator.

The main data of the system is

$\begin{array}{ll}\text { Max depth } & 100 \mathrm{~m} \\ \text { Max speed } & 3 \mathrm{knots} \\ \text { Endurance } & 4 \text { hours } \\ \text { Weight } & 150 \mathrm{~kg} \\ \text { Range } & \text { Limited by control link }\end{array}$

8.6. Teleoperation of a semi-autonomous underwater vehicle/manipulator system

A research programme has been initiated at the Center for Robotic Research at the Norwegian Institute of technology on teleoperation of a semi-autonomous underwater vehicle/manipulator system. The main idea is to have an energy-autonomous underwater vehicle with a manipulator which is controlled in telerobotic mode by an operator over a hydro-acoustic link. This means that the vehicle will be untethered, which is a great advantage when the vehicle is operating under an oil rig where cables could have limited the freedom of motion.

The hydro-acoustic link has a limited bandwidth, and in addition there is a transmission delay of $1 \mathrm{sec}$. per $1500 \mathrm{~m}$. This will obviously result in a significant time delay in the teleoperation loop which is the same problem as in teleoperation in space when the operator is on the earth (Hirzinger 1987).

A wait-and-see strategy is not satisfactory in teleoperation with time-delay, so a better solution must be developed. The two main research areas investigated in this programme is:

The use of predictive graphics using a world model generated using sensor data from the manipulator system.

The development of a stable force-reflecting interface to the operator.

Handling of unplanned impact.

\subsection{The Ocean Basin Laboratory}

The Norwegian Institute of Technology and the associated research foundation MARINTEK have a towing tank of $250 \mathrm{~m}$ and the indoor Ocean Basin Laboratory which is 80 by $50 \mathrm{~m}$ and $10 \mathrm{~m}$ deep. The Ocean Basin Laboratory has wave, current and wind generators, and the depth can be adjusted. A hydro-acoustic position measurement system with an accuracy in the range of millimetres is also installed. This laboratory facility is well suited for research on underwater operation.

\section{Road building}

Norway is a mountainous country, and tunnels are important in road construction. A computer controlled rock drilling machine was developed by the Norwegian engineering and contracting company Ingeniør Thor Furuholmen a.s. and Center for Industrial Research (SI) in Oslo, Norway (Høifødt et al. 1984). The machine has three manipulators with six joints each which makes it possible to control three drills independently in six degrees of freedom. The system is shown in Fig. 4.

This machine was introduced to the market in 1978 and has been used to drill $45 \mathrm{~mm}$ holes for many tunnels including the under-sea tunnel to the island of Vardø in Northern Norway. 


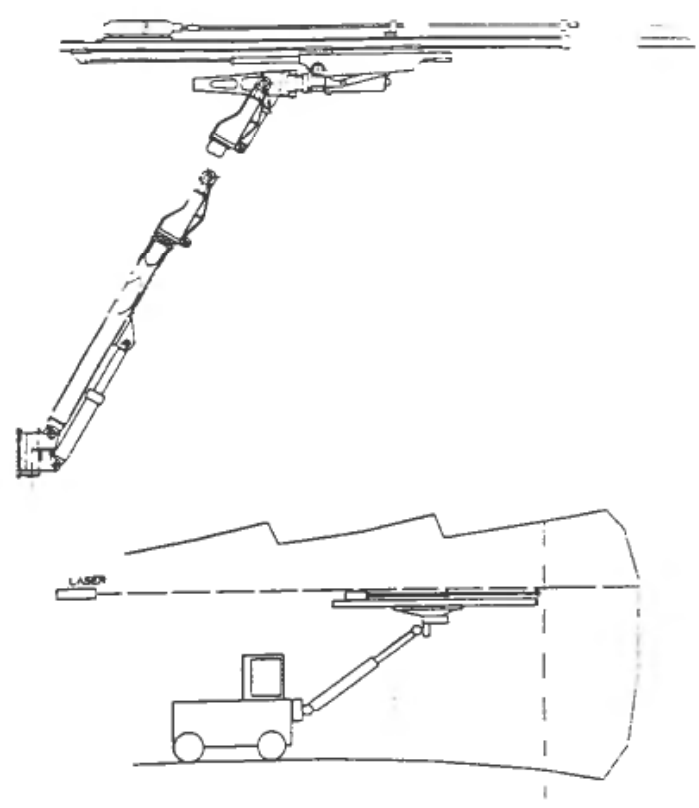

Figure 4. Automatic rock-drilling machine.

The rock drilling machine can work in joy-stick mode where the operator controls the motion of the drill in Cartesian coordinates with joy-stick, or in automatic mode where the drill pattern and the depth of the holes are defined off-line. The position is calibrated with the commonly used laser position reference system used in tunneling. The Norwegian company Bever Control a.s. has developed the control system further, and a drillplan generator developed by the Department of Civil Engineering at the Norwegian Institute of Technology is included.

The computer controlled rock drilling machine has given considerable cost savings in drilling. Improvements have also been achieved in worker conditions, precision, speed and wear on drilling equipment.

\section{REFERENCES}

ANDERSON, R. J. and SPONG, M. W.(1989). Asymptotic stability for force reflecting teleoperations with time delay, in Proc. 1989 IEEE International Conference on Robotics and Automation, Scottsdale, Arizona, May.

Egeland, O., Sagli, J. R., Hendseth, S. and Wilhelmsen, F. (1989). Dynamic coordination in a manipulator with 7 joints, in Proc. 1989 IEEE International Conference on Robotics and Automation, Scottsdale, Arizona, May.

Egeland, O., Ebdrup, M. and Chiaverini, S. (1990). Sensory control in singular configurations-Application to visual servoing, in Proc. IEEE International Workshop on Intelligent Motion Control, lstanbul, Turkey, August.

Egeland, O., Sagli, J. R., Spangelo, I. and Chiaverini, S. (1991). A damped least-squares solution to redundancy resolution, in Proc. 1991 IEEE International Conference on Robotics and Automation, Sacramento, California, April.

DickmanNS, E. D. and GRÄFE, V. (1988). (a) Dynamic monocular machine vision, (b) Application of dynamic monocular machine vision. J. Machine Vision \& Applications, 1, pp. 223-261.

Fossen, T. I. and Sagatun, S. I. (1991). Adaptive control of nonlinear underwater robotic systems, in Proc. 1991 IEEE International Conference on Robotics and Automation, Sacramento, California, April. 
HALLSET, J. O. (1991). Simple visual tracking of pipelines for an autonomous underwater vehicle, in Proc. 1991 IEEE International Conference on Robotics and Automation, Sacramento, California, April.

Hirzinger, G. (1987). The space and telerobotic concepts of DFVLR ROTEX, in Proc. 1986 IEEE International Conference on Robotics and Automation, Raleigh, North Carolina, March 1987, pp. 443-449.

HøIFøDT, J. R., WETLESEN, T. and HÅKONSEN, H. (1984). Robotics Research in Norway. Modeling, Identification and Control, 5, 151-170.

Hovland, K. (1990). Telemanipulation with force reflection (in Norwegian), MSc Thesis, Division of Engineering Cybernetics, The Norwegian Institute of Technology, Trondheim, Norway.

HusEBye, R. E., Jenssen, H. P. and Velund, D. (1990). Remotely operated subsea machine tool system, in Proc. Intervention '90 The Marine Technology Society, Vancouver, Canada, June 25 27, 1990. (Also: Report STF20 A90102, SINTEF, Trondheim, Norway.)

Kleppaker, R. A., VestgÅrd, K., Hallset, J. O. and Balchen, J. G. (1986). The application of a free-swimming ROV in aquaculture, in Proc. 9th IF AC Symposium on Automation and Dataprocessing in Aquaculture, Trondheim, Norway.

NAKAmURA, Y. and HanafuSA, H. (1986). Inverse kinematic solutions with singularity robustness for robot manipulator control, ASME J. Dynamic Syst., Meas., Contr., 108, $163-171$.

RøDSETH, Ø. J. (1990). Object oriented software system for AUV control. IARP 1st Workshop on Mobile Robots for Subsea Environments, Monterey, California, October 1990.

SAGATUN, S. I. and FOSSEN, T. I. (1991). The Norwegian experimental remotely operated vehicle (NEROV), in Proc. ROV/INTERVENTION '91, Hollywood, Florida, May 21-23.

SAGLI, J. R. and EgElAND, O. (1991). Dynamic coordination and actuator efficiency using momentum control for macro-micro manipulators, in Proc. 1991 IEEE International Conference on Robotics and Automation, Sacramento, California, April.

Sivertsen, O. I., Aamnes, K. and RølvÅg, T. (1990). General design tool for flexible space mechanisms, in C. L. Kirk and J. L. Junkins (Editors). Dynamics of Flexible Structures in Space, Proc. of the First International Conference, Cranfield, UK, May 1990, SpringerVerlag, Berlin.

Skofteland, G. and HiRzINGER, G. (1991). Computing position and orientation of a freeflying polyhedron from 3D data, in Proc. 1991 IEEE International Conference on Robotics and Automation, Sacramento, California, April.

ThOMESSEN, T. and LIEN, T. K. (1990). Grinding strategies based on contact force measurements, in Proc. 21th ISIR, Copenhagen, Denmark, October.

WhITNEY, D. E. (1987). Historic perspective and state of the art in robot force control. Int. J. Robotics Research, 6, 3-14. 